\title{
Effects of Zoledronic Acid on Bone Structure and Organization of Nanocomposites in Rats with Glucocorticoid-Induced Osteoporosis
}

\author{
Nazar Kostyshyn ${ }^{1, * \mathbb{D}}$, Liubov Kostyshyn ${ }^{1 \mathbb{D}}$, Igor Shtablavyi ${ }^{2}$ (i) \\ 1 Danylo Halytsky Lviv National Medical University, Pekarska 69, Zip Code: 79010, Lviv, Ukraine; \\ kostyshyn.nm@gmail.com(N.K.); \\ 2 Ivan Franko National University of Lviv, 6 Kyryla and Mefodia, Zip Code: 79005, Lviv, Ukraine; \\ ihor.shtablavyi@1nu.edu.ua (I.S.); \\ * Correspondence: kostyshyn.nm@gmail.com (N.K.);
}

Scopus Author ID 57204682639

Received: 12.05.2021; Revised: 4.06.2021; Accepted: 7.06.2021; Published: 18.06.2021

\begin{abstract}
Continuous use of methylprednisolone (Mps) is one of the most common causes of secondary osteoporosis resulting in alterations of the skeleton microstructure and increased fracture risk. The aim of the research was to assess the influence of zoledronic acid on mineral mass, micro- and nanostructure of the bones in rats, which received glucocorticoids. Wistar rats were randomly divided into three groups: vehicle control, the introduction of methylprednisolone, $3 \mathrm{mg} / \mathrm{kg} / \mathrm{day}$ (Mps), and Mps combined with zoledronic acid, $0.025 \mathrm{mg} / \mathrm{kg}$ (Mps+Zol.). The relative amount of a crystalline component and collagen in the bones was detected by means of X-ray diffraction, microstructure, and calcium level through atomic absorption spectroscopy. Methylprednisolone caused a decrease in the amount of a mineral component in the femoral neck (to -39.8\%) in group Mps than vehicle control. It should be emphasized that the introduction of Zol. did not allow a significant decrease in a mineral component of the bone during the experiment $\left(-11.7 \%\right.$ on the $24^{\text {th }}$ day) compared with Mps group, and indices were almost at the same level as in the control group. Zoledronic acid can partially inhibit harmful consequences of glucocorticoids for bone structure in rats and improve mineralization without impairment of crystal parameters of hydroxyapatite lattice.
\end{abstract}

Keywords: glucocorticoids; zoledronic acid; bone remodeling; bone mineral density; osteoporosis; bone nanocomposites; X-ray diffraction.

Abbreviations: BMD - bone mineral density; Zol. - zoledronic acid; Mps - Methylprednisolone sodium succinate; GCS - Glucocorticoids.

(C) 2021 by the authors. This article is an open-access article distributed under the terms and conditions of the Creative Commons Attribution (CC BY) license (https://creativecommons.org/licenses/by/4.0/).

\section{Introduction}

Osteoporosis is one of the most common diseases worldwide, which results in the reduction of bone mineral mass and increase in bone fragility and significantly increases the risk of fractures. Long-lasting treatment with medicines, in particular glucocorticoids (Gcs), is the most common cause of secondary osteoporosis [1-3], and the risk of fractures increases by $30-50 \%$ due to the reduction of the mineral density of bone tissue [4]. To model glucocorticoidinduced osteoporosis, methylprednisolone was chosen, as it is referred to as steroids and is widely used to treat inflammatory and autoimmune diseases [5,6]. The mechanism of loss of the crystalline bone component in glucocorticoid-induced osteoporosis has not been studied completely. At the cellular level, excess of glucocorticoids negatively affects bone cells, 
inhibiting osteoblasts, and launches apoptosis of osteocytes [7]. Thus, it is necessary to study bone structure not only on X-ray in vivo but also its structure at micro- and nanolevels $[8,9]$.

As it has been mentioned, bisphosphonates, in particular, zoledronic acid, are widely used in medical practice in osteoporosis treatment since it has high efficacy for inhibiting bone resorption and can be used with long intervals [12,13]. However, its administrations have certain restrictions associated with continuous use, poor tolerance, and absorption in the gastrointestinal tract. These adverse effects can be avoided by introducing it parenterally $[10,11]$. In our model, we studied the influence of zoledronic acid introduced parenterally on the density of bone tissue and bone metabolism in rats, which were injected methylprednisolone for a long period.

The main aim of our investigation was to assess the influence of osteoclast blocker zoledronic acid - on microstructure and mineral mass of the bone, modified due to glucocorticoids. A profound understanding of the nanostructure of the trabecular and cortical layers of bone tissue can highlight the efficacy of treatment with glucocorticoids and provide the possibility of using efficient therapy in osteoporosis treatment.

\section{Materials and Methods}

\subsection{Animal model and experimental design.}

The experimental study was performed on 54 male rats of the Wistar line weighing 180$200 \mathrm{~g}$. All animal experiments were conducted in compliance with bioethical principles per the provisions of the European Convention for the Protection of Vertebrate Animals used for Experimental and Other Scientific Purposes. Ethics Commission Report № 10, 16.12.2019, LNMU. The experimental rats were divided into 3 groups, 18 rats in each: vehicle control group (Veh.) - standard vivarium conditions, I experimental group - methylprednisolone sodium succinate (Mps), II experimental group - methylprednisolone sodium succinate + Zoledronic acid (Mps+Zol.). Rats were kept in the same conditions (temperature and light); water and food were available ad libitum. After the $8^{\text {th }}$, the $16^{\text {th }}$ and the $24^{\text {th }}$ week, six animals from each group were removed from the experiment by decapitation under general intraperitoneal anesthesia at $0.3 \mathrm{~g} / \mathrm{kg}$.

Methylprednisolone sodium succinate (SOLU MEDROL ${ }^{\circledR}$ ) was administered subcutaneously at a dose of $3 \mathrm{mg} / \mathrm{kg}$ every other day for 24 weeks. The vehicle control group was injected subcutaneously with $0.3 \mathrm{ml}$ of $0.9 \% \mathrm{NaCl}$. Zoledronic acid was administered at a dose of $0,025 \mathrm{mg} / \mathrm{kg}(0.1 \mathrm{ml} \mathrm{ZOLTA} ®+0.9 \mathrm{ml} 0.9 \% \mathrm{NaCl})$ intramuscularly (quadriceps femoris). Such a route of administration was chosen due to technical availability and the slow distribution of the drug. Injections were started from 4 weeks after ovariectomy, every 4 weeks until 20 weeks (remainder of the injection). No changes were observed in the injection site on the skin and subcutaneous tissue [14].

\subsection{X-ray diffraction.}

To study the ultrastructure of the tibia bone mineral component, we used the method of $\mathrm{X}$-ray diffraction analysis. The femur was dried at $110^{\circ} \mathrm{C}$ in a drying cabinet. The X-ray diffraction spectra of the samples were obtained on an automated X-ray diffractometer in $\mathrm{Cu}$ K $\alpha$ radiation $(\lambda=1.5418 \AA$ ), monochromatized by reflection from a plane (002) of a single pyrographic crystal, mounted on a diffracted beam [15-20]. We used the Bragg-Brentano focusing scheme $(\theta-2 \theta)$. The diffraction patterns were recorded in the continuous movement 
mode of the detector with an angular velocity of $2 \% \mathrm{~min}$, a constant value of the integration time $\tau=1 \mathrm{~s}$., $\mathrm{X}$-ray tube voltage at $\mathrm{U}=26 \mathrm{kV}$, and anode current at $\mathrm{I}=15 \mathrm{~mA}$.

The volume fraction of the amorphous phase was determined by the formula:

$$
\mathrm{X}=\mathrm{Ia} / \mathrm{I}
$$

where Ia - the integral intensity of the amorphous phase, I - total integrated intensity.

The average crystallite size was determined by the Debye-Scherer formula by expanding the diffraction maximum (002): $\mathrm{L}=\lambda /(\beta \cos (\theta)) ; \lambda=0,15148 \mathrm{~nm}-\mathrm{X}$-ray wavelength, $\beta$ - physical half-width of the maximum (002), $2 \theta$ - the reflection diffraction angle (002) of the crystalline phase.

\subsection{Atomic absorption spectrometric investigation.}

To determine calcium in bone tissue, samples were prior mineralized by dry ashing method with further acid extraction. Element composition was determined in prepared samples using atom-absorption spectroscopy on a device C-115PC (Academprybor, Ukraine) using acetyl/air mixture. The investigated sample was sprayed in the flame, where cold atom vapor is formed, through which light beams of the resonant frequency of calcium pass. Electrons of external membrane take in a part of a light beam, the further intensity of which is determined by a detector, and it is proportional to the concentration of the element in flame.

\subsection{Field emission scanning electron microscopy.}

To investigate the microstructure of the bone samples, a field emission scanning electron microscopy (FESEM) analysis was performed using a Hitachi S-4100 microscope with a secondary electron detector. The scanning of the sample surface was carried out by electron beam operating at $15 \mathrm{kV}$ and $10 \mu \mathrm{A}$ with the spatial resolution of $100 \mathrm{~nm}$ in the secondary electron image regime [21-23].

\subsection{Statistical analysis.}

Statistical analysis of the data was performed in StatSoft STATISTICA 8.0.360. In the STATISTICA package, the comparison of two average samples of normally distributed features (Student's t-criterion) was implemented in the Basic Statistics/Tables module. The ttest, independent, by variable submodule, was used for two different general summations.

\section{Results and Discussion}

The quantitative composition and the correlation between crystalline and amorphous phases of the bone were determined by diffuse halo on small angles of diffraction, particularly in the region of angles $2 \theta=15-37.5^{\circ}$. The diffraction pattern was distributed into discrete maximums, which correspond to diffraction with amorphous and crystalline phases of the bone. Diffraction patterns of bone hydroxyapatite powder are presented in Figure 1(a) [20]. Shown peaks represent diffraction form crystal lattices. The periodic arrangement of atoms in mineral crystals and the distribution of overlap regions of gaps in collagen fibers enables the use of Xray diffraction to investigate bone nanostructure in the experiment. 


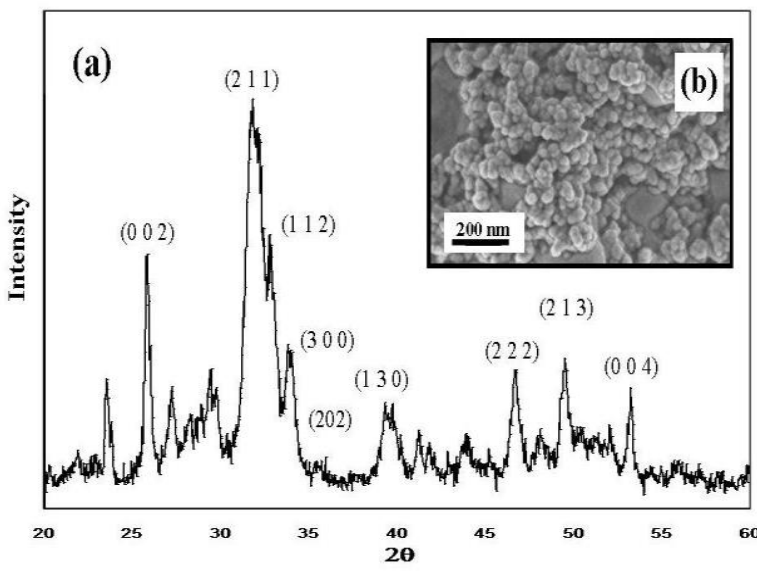

(a)

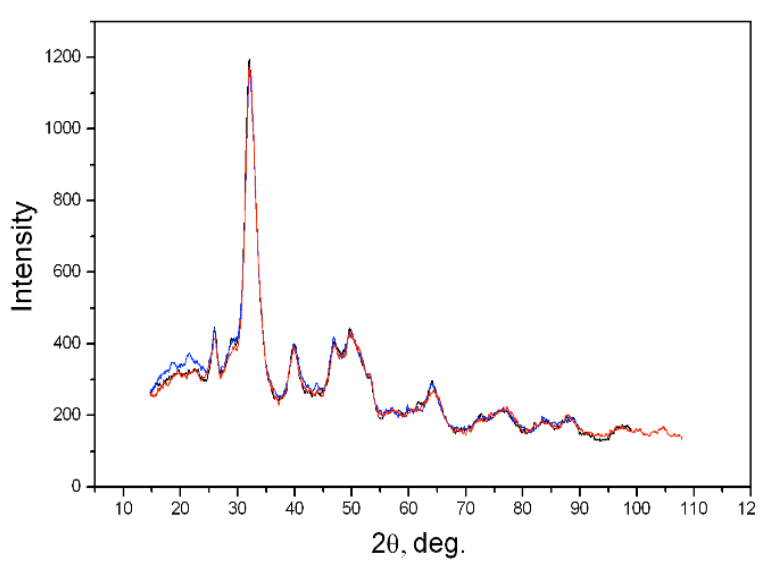

(b)

Figure 1. The diffraction spectra of the samples were obtained on an automated X-ray diffractometer in $\mathrm{Cu} \mathrm{K} \alpha$ radiation by monochromatic reflection from the plane (002) of a single graphite crystal mounted on a diffracted beam. Although this reflex consists of four reflexes of hydroxyapatite with Miller indices (211); (112); (300);

(202), the diffraction angles from crystallographic planes with these indices are close to $31,741^{0} ; 32,179^{0}$; 32,868 , and 34,045. Typical experimental powder XRD spectrum of hydroxyapatite (Hap.) sample [20], (a-(a)

FESEM micrograph showing the spherical morphology of $30 \pm 5 \mathrm{~nm}$ Hap. particles (a-(b). On the spectra of samples (b) in the sequence 8 weeks $\rightarrow 16$ weeks $\rightarrow 24$ weeks, the volume fraction of the crystalline phase does not change in rats of the control group.

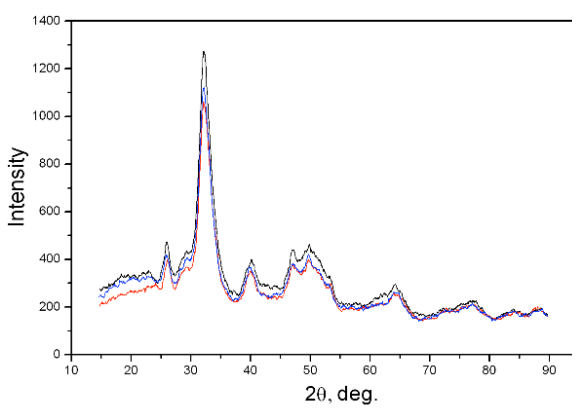

(a)

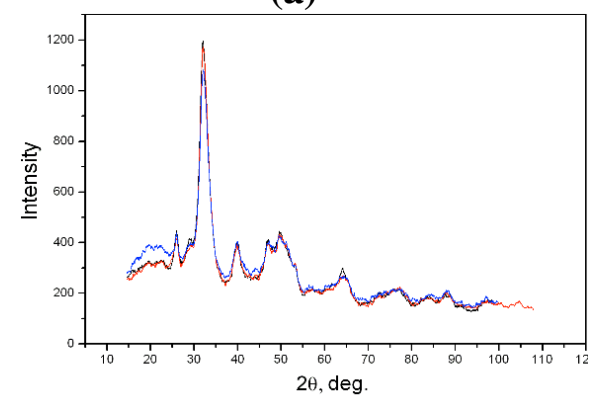

(d)

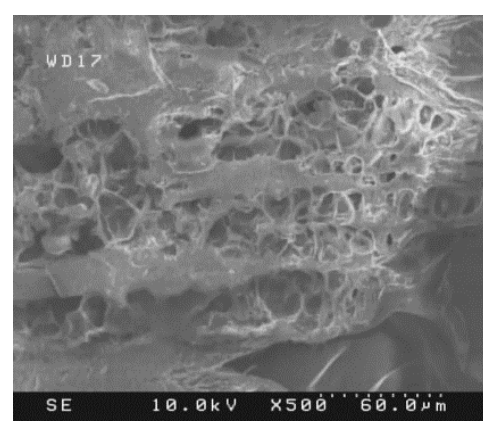

(b)

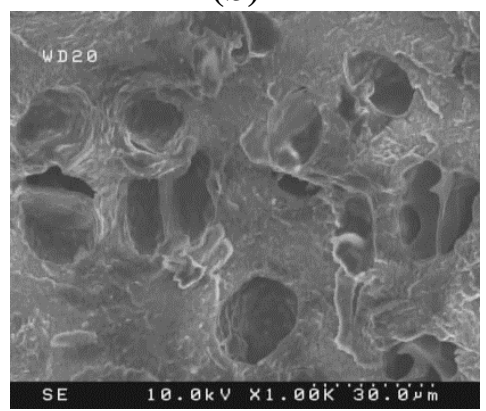

(e)

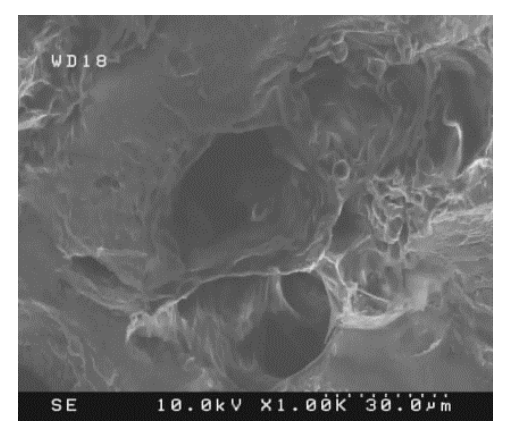

(c)

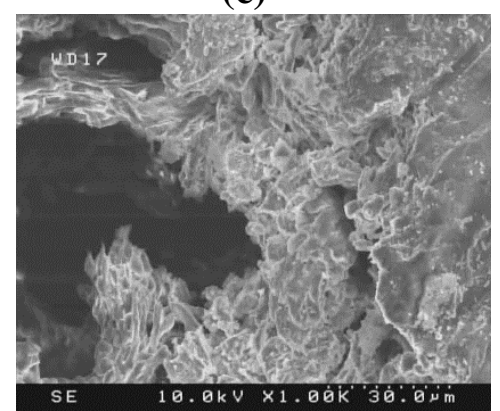

(f)

Figure 2. Diffractograms (a) and electron microscopy of samples of the femoral neck in the group Mps (b, c) in group Mps+Zol $(\mathbf{d}, \mathbf{e}, \mathbf{f})$ on $-8^{\text {th }} ;-16^{\text {th }} ;-24^{\text {th }}$ week of investigation.

Peaks, which present areas of lattices, are characterized depending on their distribution, crystallinity, and order of crystals. However, mineral particles in the bone are different forms of apatite, particularly hydroxyapatite, which has lower crystallinity. Thus, distinct peaks are absent in diffraction profiles of bone mineral crystals, which possess characteristics close to apatite crystals, Figure 1a,b [20].

Application of X-ray structure analysis combined with scanning electron microscopy enabled, in addition to revealing the nature of the mineral phase of the bones, to study the 
structure of the main protein component of the bone - collagen. Spectral extrapolation of data indicates the tendency to increase in the amount of amorphous phase almost in all samples; however, in experimental group I, this value is the highest, indicating accelerated bone remodeling and rapid loss of a mineral component under the influence of steroid hormones. Scanning electron microscopy enabled the assessment of microarchitecture characteristics of samples in terms of morphological description of osteocyte lacunae and geometry of canals. Figure 2 presents cross-sections of the trabecular layer with osteocyte lacunae and trabeculae. The microstructure of the femoral neck in Mps group on the 24th day of the experiment indicates widening of bone canals and thinning of the trabeculae. Numerous lacunae of bone resorption and dilation of nervous and vascular canals are found on the surface of the trabecular bone in experimental group I. Microstructure of the femoral neck in group Mps+Zol on the 24th day of the experiment indicates the arrangement of the structure with the common direction of collagen fibers, which are located in parallel layers, forming bone plates. Areas of restoration are detected on the surface of bone cross-sections in experimental group II (Mps+Zol), where fewer lacunae of resorption are found compared with experimental group I, and some nervous and vascular canals are slightly dilated.

Table 1. Volume fraction of amorphous phase and size of crystallites.

\begin{tabular}{l|l|l|l|l} 
Group/week & Xa, (r.i.) & Ia & $\mathbf{I}$ & $\mathbf{L}, \mathbf{n m}$ \\
\hline Vehicle 8 week & $0.209 \pm 0.018$ & 806 & 3862 & $13.7 \pm 0.5$ \\
\hline Vehicle 16 week & $0.190 \pm 0.025$ & 695 & 3667 & $13.5 \pm 0.6$ \\
\hline Vehicle 24 week & $0.215 \pm 0.025$ & 624 & 2901 & $13.0 \pm 0.6$ \\
\hline Mps 8 week & $0.211 \pm 0.034$ & 876 & 4159 & $13.6 \pm 0.7$ \\
\hline Mps 16 week & $0.215 \pm 0.017$ & 655 & 3041 & $11.1 \pm 0.5$ \\
\hline Mps 24 week & $0.231 \pm 0.049$ & 871 & 3773 & $12.4 \pm 0.6$ \\
\hline Mps+Zol. 8 week & $0.179 \pm 0.017$ & 511 & 2860 & $12.1 \pm 0.5$ \\
\hline Mps+Zol. 16 week & $0.167 \pm 0.011$ & 582 & 3481 & $14.6 \pm 0.4$
\end{tabular}

$\mathrm{Xa}$ - volume fraction of the amorphous phase; Ia - the integral intensity of the amorphous phase; $\mathrm{I}$ - total integrated intensity; $\mathrm{L}$ - the average crystallite size of the $\mathrm{Ca}_{10} \mathrm{P}_{6} \mathrm{O}_{26} \mathrm{H}_{2}$ phase;

* - groups differ statistically, $\mathrm{p}<0.05$ (Control vs Mps);

** - groups differ statistically, $\mathrm{p}<0.05$ (Mps vs Mps+Zolta).

In experimental group I (Mps), a statistically significant reduction of hydroxyapatite amount is observed already during the $8^{\text {th }}-16^{\text {th }}$ weeks, and partial stabilization of this process occurs by the $24^{\text {th }}$ week; relative amount of hydroxyapatite in these groups decreased to $17 \%$ by the end of the experiment. An increase in the amorphous phase (collagen) volume is also noticed, which indicates accelerated remodeling. In rats, which were introduced methylprednisolone and zoledronic acid, higher values of crystalline phase were observed compared with indices in the control group during the same period. Zoledronic acid prevented both early losses of a mineral component after the continuous introduction of methylprednisolone for 8-16 weeks and at the end of the experiment. Impact of Zol. on rats, which were injected glucocorticoids during the $8^{\text {th }}-16^{\text {th }}$ weeks, indicated a slight decrease in the volume of a crystalline component, which was by $6-10 \%$ lower than in the animals receiving placebo $(\mathrm{p}<0.05)$ and by $17 \%$ higher than in the animals treated with glucocorticoids $(\mathrm{p}<0.05)$ (Figure. 2, 3; Table 1). The charts show that collagen metabolism increases in group $\mathrm{I}$ in addition to the aforementioned effects, which demonstrates accelerated remodeling and explains the rapid loss of BMD. Damage to the crystal lattice of hydroxyapatite was not observed in all experimental groups (Table 1). These phenomena are confirmed by electron 
microscopy, where areas of bone tissue mineralization due to Zol. are observed, and in Mps group - thinning of trabeculae. However, the application of X-ray structure analysis and electron microscopy for the assessment of a trabecular bone layer does not allow assessing loss of bone mass completely. Thus, we used atomic absorption spectrometry to determine the amount of calcium in investigated bone fragments (femoral neck).

During the $8^{\text {th }}$ week of the experiment, a decrease in calcium was observed in the samples of the first experimental group by $17 \%$ compared with the control group (Table 2 ). In the second experimental group, a decrease in quantitative indices of calcium was also recorded, but this value was $9 \%$ lower than in the control group. During the $16^{\text {th }}$ and $24^{\text {th }}$ weeks of monitoring, calcium mass reduced only in the first experimental group by $-38.4 \%$ and by 39.8 $\%$ compared with the control group ( $\mathrm{p}<0.05)$. It likely indicates the continuation of bone remodeling with high activity of osteoclasts and osteoblasts, which results in loss of mineral mass of the femoral bone. In experimental group II (Mps+Zol), an increase in mineral mass of calcium constituted $+37.3 \%$ compared with experimental group I during the $16^{\text {th }}$ week of the experiment and $+31.8 \%$ during the $24^{\text {th }}$ week. Besides, calcium loss at $-11.7 \%$ was also observed during the $24^{\text {th }}$ week in experimental group II; however, this index was statistically insignificant compared with the control group.

Table 2. Content of calcium $(\mathrm{mg} / \mathrm{g})$ in fragments of the femoral neck in rats.

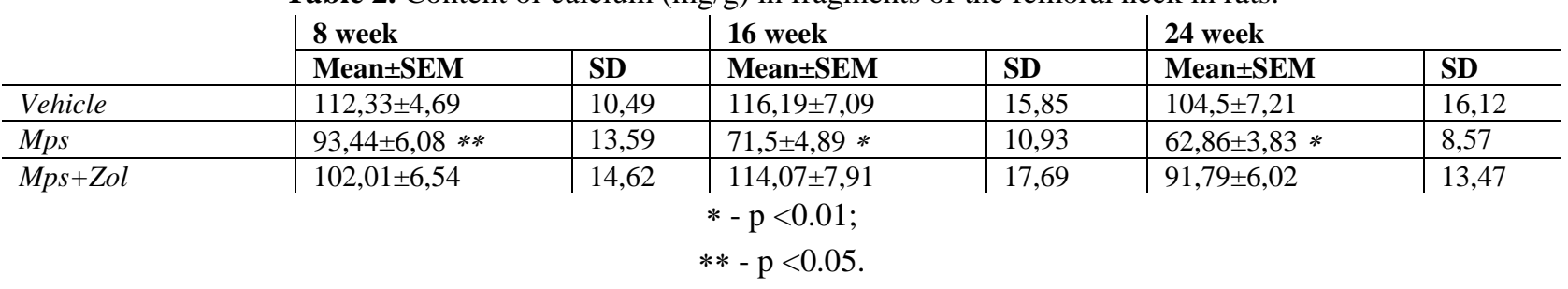

\subsection{Discussion.}

We have established that zoledronic acid can inhibit some side effects of glucocorticoids on bone structure in rats and their metabolism. Continuous intake of glucocorticoids significantly reduces the amount of a crystalline component in bone tissue, which likely results in thinning of trabeculae. However, in rats of experimental group II, which were injected zoledronic acid and were subjected to the side effect of methylprednisolone, crystal parameters of their bones did not differ considerably until the $16^{\text {th }}$ week of the experiment. It enabled to preserve more mineral mass by 31.8 - 37.3\% compared with quantitative indices in experimental group I. We have conducted an assessment of bone structure and its organization at micro- and nano levels, which can help understand mechanisms of loss of bone mineral mass that lead to alterations in its properties as strength and resilience. Due to non-destructive properties, X-rays are widely used to examine crystalline materials, but they are rarely used to study biological tissues, particularly bone. It would enable better understanding of the structural composition of their molecular components.

$\mathrm{X}$-ray diffraction combined with scanning electron microscopy proved an efficient technique to study structural and mechanical characteristics of bone minerals and assess the condition of crystallites and the amount of amorphous component in the composition, which can be directly associated with phenomena of crystallites bone tissue remodeling and adaptation. This method helps detect local deformations of crystal lattices of hydroxyapatite in vitro in certain regions of bone tissue. However, the orientation of mineral crystals and 
distribution of their volumetric fraction differs due to the influence of glucocorticoids, which is an important factor regulating peculiarities of the structure of nanosized materials.

Our earlier investigations conducted a correction of bone tissue condition via the additional impact of non-physiological vibration with acceleration $0.3 \mathrm{~g}$ under conditions of obesity, consumption of fructose, deficiency of sex hormones, and influence of glucocorticoids. We also established that vibration has an anabolic influence on the skeleton and can prevent the loss of mineral mass $[24,25]$.

However, in addition to studying the loss of bone tissue mineral components, diagnostics of protein fibers is also important. A combination of these components forms a natural nanocomposite. It is understandable that not only bone density determines its strength in general. The femoral neck was used in this investigation since many pathological fractures occur in this region. Information on the basic structural unit of the bone mineral collagen composite provides a better understanding of bone tissue's volumetric structure and strength. The introduction of glucocorticoids statistically significantly reduced the amount of crystalline components and calcium. Zoledronic acid had a positive impact on bone tissue both during the $8^{\text {th }}$ week of the experiment and after continuous treatment with glucocorticoids, and during the $24^{\text {th }}$ week, this influence was statistically significant. We determined that zoledronic acid considerably increased the mineral mass of a trabecular layer of the femoral neck in rats, which received glucocorticoid therapy. The efficacy of Zol. did not differ significantly according to the prior duration of glucocorticoid therapy, which confirms its administration both for prevention and treatment of osteoporosis caused by glucocorticoids. Unexpectedly, it proved that the application of zoledronic acid is efficient not only for rats, which received methylprednisolone at the onset of the experiment, but also during the 24th week of the investigation loss of bone mass was observed compared with the control group.

\section{Conclusions}

In our investigations, deceleration of remodeling and, as a result, decrease in the loss of bone tissue mineral component corresponds to the results of other investigations on zoledronic acid efficacy. It provides additional proofs that this bisphosphonate decelerates bone resorption and can be used for treatment and prevention of glucocorticoid-induced osteoporosis. Thus, we assume that our experimental model can be implemented for patients who receive continuous therapy with glucocorticoids.

\section{Funding}

This research received no external funding.

\section{Acknowledgments}

The authors would like to acknowledge the University of Wurzburg for providing the equipment for the FESEM investigation.

\section{Conflicts of Interest}

The authors declare no conflict of interest. 


\section{Ethics approval and consent to participate}

\section{Ethics Commission Report №10, 16.12.2019, Danylo Halytsky Lviv National Medical} University, Lviv, Ukraine.

\section{References}

1. Bhattarai, H.K.; Shrestha, S.; Rokka, K., Shakya, R. Vitamin D, Calcium, Parathyroid Hormone, and Sex Steroids in Bone Health and Effects of Aging. Journal of Osteoporosis 2020, https://doi.org/10.1155/2020/9324505.

2. Li, R.; Wen, Z.G.; Li, H.X.; Yu, Q.; Xu, Y.; \& Xiao, S.M. Associations of Sex Steroids With Changes in Calcaneal Quantitative Ultrasound Measurements: A Longitudinal Study in Chinese Male Adolescents. Journal of $\quad$ Clinical Densitometry $\quad \mathbf{2 0 2 0}, \quad 23, \quad 588-595$, https://doi.org/10.1016/j.jocd.2020.01.001.

3. Khosla, S.; Monroe, D.G. Regulation of bone metabolism by sex steroids. Cold Spring Harbor perspectives in medicine 2018, 8, a031211, https://doi.org/10.1101/cshperspect.a031211.

4. Briot, K.; Roux, C. Glucocorticoid-induced osteoporosis. RMD open 2015, 1, e000014, https://doi.org/10.1136/rmdopen-2014-000014.

5. Hu, K.; Adachi, J.D. Glucocorticoid induced osteoporosis. Expert Rev Endocrinol Metab 2019, 14, 259-266, https://doi.org/10.1080/17446651.2019.1617131.

6. Morgan, H.; Chan, C.; Clynes, M.; Jameson, K.; Holroyd, C.; Kate Ward; Mark Edwards; Cooper, C.; Dennison, E Bone Health in Rheumatoid Arthritis: What Can Studies of Bone Microarchitecture Tell Us? Rheumatology 2020, 7, 91-99.

7. Zelikovich, A.S.; Quattrocelli, M.; McNally, E.M.; Kuntz, N.L. Improved Metabolic and Bone Health in Boys with Duchenne Muscular Dystrophy Treated with High Dose Pulse Weekend Corticosteroids compared to Daily Dosing. Neurology 2020, 94, 21, https://n.neurology.org/content/94/15_Supplement/21.

8. Sanchez-Siles, J.M.; Tamimi-Mariño, I.; Cortes, A.G.; Ackerman, J.L.; González-Quevedo, D.; Guerado, E.; Tamimi, F. Age related changes in the bone microstructure in patients with femoral neck fractures. Injury 2020, 51, 12-18, https://doi.org/10.1016/j.injury.2020.02.014.

9. Nakajima, K.; Matsunaga, S.; Morioka, T.; Nakano, T.; Abe, S.; Furuya, Y.; Yajima, Y. Effects of unloading by tail suspension on biological apatite crystallite alignment in mouse femur. Dental Materials Journal 2020, 39, 670-677, https://doi.org/10.4012/dmj.2019-187.

10. You, R.; Zhang, Y.; Wu, D.C.; Liu, J.; Qian, X.; Luo, N.; Mori, T. Cost-effectiveness of zoledronic acid versus oral alendronate for postmenopausal osteoporotic women in China. Frontiers in Pharmacology 2020, 11, 456, https://doi.org/10.3389/fphar.2020.00456.

11. Pozzi, S.; Vallet, S.; Mukherjee, S.; Cirstea, D.; Vaghela, N.; Santo, L.; Rosen, E.; Ikeda, H.; Okawa, Y.; Kiziltepe, T.; Schoonmaker, J.; Xie, W.; Hideshima, T.; Weller, E.; Bouxsein, M.L.; Munshi, N.C.; Anderson, K.C.; Raje, N. High-dose zoledronic acid impacts bone remodeling with effects on osteoblastic lineage and bone mechanical properties. Clinical Cancer Research 2009, 15, 5829-5839, https://doi.org/10.1158/10780432.CCR-09-0426.

12. Boran, G.; Tavakoli, S.; Dierking, I.; Kamali, A. R.; Ege, D. Synergistic effect of graphene oxide and zoledronic acid for osteoporosis and cancer treatment. Scientific reports 2020, 10, 1-12, https://doi.org/10.1038/s41598-020-64760-4.

13. Huang, S.Y.; Yoon, S.S.; Shimizu, K.; Chng, W.J.; Chang, C.S.; Wong, R.S. M.; Min, C.K. Denosumab Versus Zoledronic Acid in Bone Disease Treatment of Newly Diagnosed Multiple Myeloma: An International, Double-Blind, Randomized Controlled Phase 3 Study-Asian Subgroup Analysis. Advances in Therapy 2020, 37, 3404-3416, https://doi.org/10.1007/s12325-020-01395-X.

14. Kostyshyn, N.M.; Gzhegotskyi, M.R.; Kostyshyn L.P.; Mudry, S.I. Effect of zoledronic acid on bone nanocomposites organization and prevention of bone mineral density loss in ovariectomized rats. Drug Metabolism and Personalized Therapy 2021. https://doi.org/10.1515/dmpt-2020-0187

15. Rabiei, M.; Palevicius, A.; Monshi, A.; Nasiri, S.; Vilkauskas, A.; Janusas, G. Comparing methods for calculating nano crystal size of natural hydroxyapatite using X-Ray diffraction. Nanomaterials 2020, 10, 1627, https://doi.org/10.3390/nano10091627.

16. Clark S.M.; Iball, J. The X-ray crystal analysis of bone. Progress in Biophysics and Biophysical Chemistry: Progress Series 2016, 7, 226, https://doi.org/10.1016/S0096-4174(18)30127-6. 
17. Bunaciu, A.A.; UdriŞTioiu, E.G.; Aboul-Enein, H.Y. X-ray diffraction: instrumentation and applications. Critical reviews in analytical chemistry 2015, 45, 289-299, https://doi.org/10.1080/10408347.2014.949616.

18. Londoño-Restrepo, S.M.; Herrera-Lara, M.; Bernal-Alvarez, L.R.; Rivera-Muñoz, E.M.; Rodriguez-García, M.E. In-situ XRD study of the crystal size transition of hydroxyapatite from swine bone. Ceramics International 2020, 46, 24454-24461, https://doi.org/10.1016/j.ceramint.2020.06.230.

19. Piga, G.; Solinas, G.; Thompson, T.U.; Brunetti, A.; Malgosa, A.; Enzo, S. Is X-ray diffraction able to distinguish between animal and human bones? Journal of archaeological science 2013, 40, 778-785, https://doi.org/10.1016/j.jas.2012.07.004.

20. Brundavanam, R.K.; Poinern, G.J.; Fawcett, D. Modelling the crystal structure of a $30 \mathrm{~nm}$ sized particle based hydroxyapatite powder synthesised under the influence of ultrasound irradiation from X-ray powder diffraction data.American Journal of Materials Science 2013, 3, 84-90, https://doi.org/10.5923/j.materials.20130304.04.

21. Shah, F.A.; Ruscsák, K.; Palmquist, A. 50 years of scanning electron microscopy of bone-a comprehensive overview of the important discoveries made and insights gained into bone material properties in health, disease, and taphonomy. Bone research 2019, 7, 1-15, https://doi.org/10.1038/s41413-019-0053-z.

22. Alexander, B.; Daulton, T.L.; Genin, G.M.; Lipner, J.; Pasteris, J.D.; Wopenka B.; Thomopoulos, S. The nanometre-scale physiology of bone: steric modelling and scanning transmission electron microscopy of collagen-mineral structure. Journal of the Royal Society Interface 2012, 9, 1774-1786, https://doi.org/10.1098/rsif.2011.0880.

23. Boyde, A. Scanning electron microscopy of bone. In Bone Research Protocols. Humana Press, New York, 2019, 571-616, https://doi.org/10.1007/978-1-4939-8997-3_31.

24. Kostyshy, N.; Kulyk, Y.; Kostyshyn, L.; Gzhegotskyi, M. Metabolic and Structural Response of Bone to Whole-Body Vibration in Obesity and Sedentary Rat Models for Osteopenia. Romanian Journal of Diabetes Nutrition and Metabolic Diseases 2020, 27, 200-208, https://doi.org/10.46389/rjd-2020-1031.

25. Kostyshyn, N.M.; Gzhegotskyi. M.R.; Kostyshyn, L.P.; Mudry, S.I. Effects of mechanical stimuli on structure and organization of bone nanocomposites in rats with glucocorticoid-induced osteoporosis. Endocrine Regulations 2021, 55, 42-51, https://doi.org/10.2478/enr-2021-0006. 